Rafail Alizade · Sultan Eylem Toksoy

\title{
Cofinitely Supplemented Modular Lattices
}

Received: 24 October 2010 / Accepted: 6 March 2011 / Published online: 12 August 2011

(c) King Fahd University of Petroleum and Minerals 2011

\begin{abstract}
In this paper it is shown that a lattice $L$ is a cofinitely supplemented lattice if and only if every maximal element of $L$ has a supplement in $L$. If $a / 0$ is a cofinitely supplemented sublattice and $1 / a$ has no maximal element, then $L$ is cofinitely supplemented. A lattice $L$ is amply cofinitely supplemented if and only if every maximal element of $L$ has ample supplements in $L$ if and only if for every cofinite element $a$ and an element $b$ of $L$ with $a \vee b=1$ there exists an element $c$ of $b / 0$ such that $a \vee c=1$ where $c$ is the join of finite number of local elements of $b / 0$. In particular, a compact lattice $L$ is amply supplemented if and only if every maximal element of $L$ has ample supplements in $L$.
\end{abstract}

Keywords Cofinite element - Ample supplement - Amply supplemented lattice . Cofinitely supplemented lattice $\cdot$ Amply cofinitely supplemented lattice

Mathematics Subject Classification (2010) 06CXX · 16D10

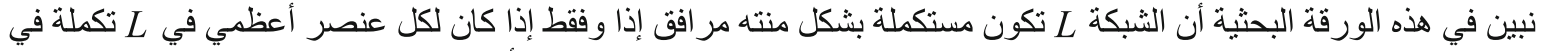

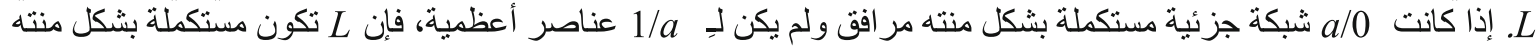

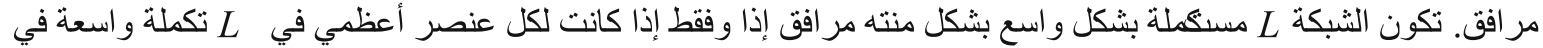

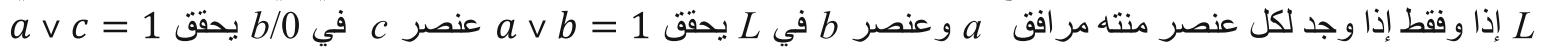

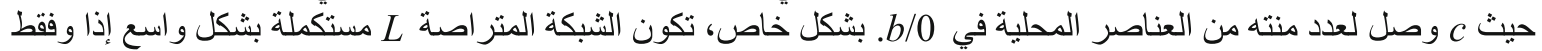

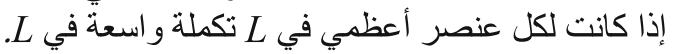

\section{Introduction}

Throughout $L$ denotes an arbitrary complete modular lattice with smallest element 0 and greatest element 1 . A sublattice of the form $b / a=\{x \in L \mid a \leq x \leq b\}$ is called a quotient sublattice [3]. An element $a$ of a

R. Alizade $(\varangle)$

Department of Mathematics, Yaşar University, Selçuk Yaşar Campus, Üniversite Caddesi, No: 35-37, Ağaçlı Yol,

35100 Bornova, Izmir, Turkey

E-mail: rafail.alizade@yasar.edu.tr

S. E. Toksoy

Department of Mathematics, Izmir Institute of Technology, 35430 Urla, Izmir, Turkey

E-mail: eylemtoksoy@iyte.edu.tr 
lattice $L$ is said to be small if $a \vee b \neq 1$ holds for every $b \neq 1$. It is denoted by $a \ll L$. We will write $a<b$ if $a \leq b$ and $a \neq b$. We have the following properties of small elements:

Lemma 1.1 [3, Lemmas 7.2, 7.3 and 12.4] Let $a<b$ be elements in a lattice L.

(1) If $a \ll b / 0$, then $a \vee c \ll(b \vee c) / c$ for every $c \in L$.

(2) $b \ll L$ if and only if $a \ll L$ and $b \ll 1 / a$.

(3) Let $c^{\prime} \ll c / 0$ and $d^{\prime} \ll d / 0$. Then $c^{\prime} \vee d^{\prime} \ll(c \vee d) / 0$.

An element $a$ of $L$ is called a supplement of an element $b$ if $a \vee b=1$ and $a$ is minimal with respect to this property. Equivalently, an element $a$ is a supplement of $b$ in $L$ if and only if $a \vee b=1$ and $a \wedge b \ll a / 0[3$, Proposition 12.1]. A lattice $L$ is said to be supplemented if every element $a$ of $L$ has a supplement in $L$. An element $a$ of a lattice $L$ is said to be cofinite in $L$ if the quotient sublattice $1 / a$ is compact, that is $1=\bigvee_{i \in I} x_{i}$ for some elements $x_{i} \geq a$ implies that $1=\bigvee_{i \in F} x_{i}$ for some finite subset $F$ of $I$. A lattice $L$ is said to be cofinitely supplemented if every cofinite element of $L$ has a supplement in $L$. In Section 2 we prove that a lattice $L$ is cofinitely supplemented if and only if every maximal element of $L$ has a supplement in $L$. Using this we show that if a lattice $L$ is an arbitrary join of cofinitely supplemented elements $a_{i}$ with $a_{i} / 0$ cofinitely supplemented, then $L$ is cofinitely supplemented. Also we prove that $L$ is cofinitely supplemented if $a / 0$ is a cofinitely supplemented sublattice and $1 / a$ has no maximal elements.

An element $a$ of a lattice $L$ has ample supplements in $L$ if for every element $b$ of $L$ with $a \vee b=1, b / 0$ contains a supplement of $a$ in $L$. A lattice $L$ is said to be amply supplemented if every element $a$ of $L$ has ample supplements in $L$. In Section 3 we generalize some properties of amply supplemented modules to amply supplemented lattices. Also we study amply cofinitely supplemented lattices, that is lattices whose cofinite elements have ample supplements. A lattice $L$ is said to be local if the set of elements different from 1 has a largest element. An element $l$ is called a local element if the quotient sublattice $l / 0$ is local. We show in Theorem 3.9 that a lattice $L$ is amply cofinitely supplemented if and only if every maximal element of $L$ has ample supplements in $L$. Moreover in this situation for every cofinite element $a$ and an element $b$ of $L$ with $a \vee b=1$ there exists an element $c$ of $b / 0$ such that $a \vee c=1$ where $c$ is the join of finite number of local elements of $b / 0$. In particular, a compact lattice $L$ is amply supplemented if and only if every maximal element of $L$ has ample supplements in $L$.

We give proofs of the results for lattices when the proofs are different from those in the module case. All definitions and related properties not given here can be found in $[3,4]$.

\section{Cofinitely Supplemented Lattices}

An element $c$ of $L$ is said to be compact, if for every subset $X=\left\{x_{i} \mid i \in I\right\}$ of $L$ with $c \leq \bigvee_{i \in I} x_{i}$ there exists a finite subset $F$ of $I$ such that $c \leq \bigvee_{i \in F} x_{i}$. A lattice $L$ is said to be compact if 1 is compact and compactly generated (or algebraic) if each of its elements is a join of compact elements [6]. For compactly generated compact lattices a supplement of an element is compact [3, Proposition 12.2 (2)]. In the following proposition we show that for an arbitrary lattice $L$ a supplement of a cofinite element is compact:

Proposition 2.1 Let a be a cofinite element of a lattice $L$ and $b$ be a supplement of $a$. Then $b / 0$ is compact.

Proof Since $b$ is a supplement of $a$ in $L, a \vee b=1$ and $b$ is minimal with respect to this property. Let $b=\bigvee_{i \in I} b_{i}$ for some $b_{i} \leq b$.

$$
1=a \vee b=a \vee\left(\bigvee_{i \in I} b_{i}\right)=\bigvee_{i \in I}\left(a \vee b_{i}\right)
$$

Since $a$ is cofinite, $1 / a$ is compact, there exists a finite subset $F$ of $I$ such that

$$
1=\bigvee_{i \in F}\left(a \vee b_{i}\right)=a \vee\left(\bigvee_{i \in F} b_{i}\right)
$$

Then $b=\bigvee_{i \in F} b_{i}$ by minimality of $b$. So $b$ is compact.

Recall that a lattice $L$ is said to be local if the set of elements different from 1 has a largest element. 
Lemma 2.2 [1, Lemma 2.9] Let $\left\{l_{i} / 0\right\}_{i \in I}, I=\{1, \ldots, n\}$ be a finite collection of local sublattices of a lattice $L$ and $a$ be an element of $L$ such that $a \vee\left(\bigvee_{i \in I} l_{i}\right)$ has a supplement $b$ in $L$. Then there exists a subset $J$ of $I$ such that $b \vee\left(\bigvee_{i \in J} l_{i}\right)$ is a supplement of $a$ in $L$.

Proof Induction on $n$. For $n=1, b$ is a supplement of $a \vee l_{1}$, i.e. $b \vee\left(a \vee l_{1}\right)=1$ and $b \wedge\left(a \vee l_{1}\right) \ll b / 0$. Put $c=(a \vee b) \wedge l_{1}$. If $c=l_{1}$, then $l_{1} \leq a \vee b$. So $1=b \vee\left(a \vee l_{1}\right)=a \vee b$ and $b \wedge a \leq b \wedge\left(a \vee l_{1}\right) \ll b / 0$. Thus $b$ is a supplement of $a$ in $L$. If $c \neq l_{1}$, then $(a \vee b) \wedge l_{1}=c \ll l_{1} / 0$. Therefore, $l_{1}$ is a supplement of $c$ in $l_{1} / 0$. By [3, Lemma 12.3 ] and Lemma 1.1 (3) the following holds:

$$
a \wedge\left(b \vee l_{1}\right) \leq\left[b \wedge\left(a \vee l_{1}\right)\right] \vee\left[l_{1} \wedge(a \vee b)\right] \ll\left(b \vee l_{1}\right) / 0
$$

So $b \vee l_{1}$ is a supplement of $a$. Suppose that $n>1$ and $b$ is a supplement of $a^{\prime} \vee\left(\bigvee_{i=2}^{n} l_{i}\right)$ in $L$ where $a^{\prime}=a \vee l_{1}$. By the induction hypothesis there is a subset $I^{\prime}$ of $\{2, \ldots, n\}$ such that $b^{\prime}=b \vee\left(\bigvee_{i \in I^{\prime}} l_{i}\right)$ is a supplement of $a^{\prime}=a \vee l_{1}$, by the case $n=1$ either $b^{\prime}$ or $b^{\prime} \vee l_{1}$ is a supplement of $a$ in $L$. This completes the proof.

Lemma 2.3 [3, Lemma 12.5 (b)] In a lattice $L$ let $m$ be a maximal element. If $l$ is a supplement of $m$, then $l / 0$ is local. Moreover, $l \wedge m$ is the largest element of $l / 0$ different from $l$.

Proof $l$ is a supplement of $m$ if and only if $l \vee m=1$ and $l \wedge m \ll l / 0$. Let $x \in l / 0$ and $x \neq l$. If $x \leq m$, then $x \leq l \wedge m$. If $x \not \leq m(x \not \leq l \wedge m)$, then since $m$ is maximal $x \vee m=1$.

$$
l=l \wedge 1=l \wedge(x \vee m)=x \vee(l \wedge m) .
$$

Since $l \wedge m \ll l / 0, x=l$. This is a contradiction. Thus $l \wedge m$ is the largest element $(\neq l)$ of $l / 0$.

Theorem 2.4 [5, Theorem 5.3.33] A lattice $L$ is a cofinitely supplemented lattice if and only if every maximal element of $L$ has a supplement in $L$.

Proof $(\Rightarrow)$ Let $m$ be a maximal element of $L$. Then there are only two elements of $1 / m: 1, m$. So $m$ is cofinite. Since $L$ is cofinitely supplemented, $m$ has a supplement in $L$.

$(\Leftarrow)$ Let the join of local elements of $L$ be denoted by $\operatorname{Loc}(L)$. Let $m$ be a maximal element in $1 / \operatorname{Loc}(L)$. Then $m$ is a maximal element of $L$. By assumption $m$ has a supplement $b$ in $L$. By Lemma $2.3, b / 0$ is a local sublattice; therefore, $b$ is a local element. Then $b \leq \operatorname{Loc}(L) \leq m$ and so $1=m \vee b=m$. This is a contradiction. So there is no maximal element in $1 / \operatorname{Loc}(L)$. Let $a$ be a cofinite element of $L$. Then $a \vee \operatorname{Loc}(L)$ is cofinite in $L$. Since there is no maximal element in $1 / \operatorname{Loc}(L), 1 /(a \vee \operatorname{Loc}(L))$ has no maximal element, but by [3, Lemma 2.4], if $a \vee \operatorname{Loc}(L) \neq 1$, then $1 /(a \vee \operatorname{Loc}(L))$ has at least one maximal element $(\neq 1)$. So $a \vee \operatorname{Loc}(L)=1$. Since $1 / a$ is compact for some local elements $l_{1}, \ldots, l_{n}$ of $L$

$$
a \vee\left(l_{1} \vee \cdots \vee l_{n}\right)=1 .
$$

0 is a supplement of $a \vee\left(l_{1} \vee \cdots \vee l_{n}\right)=1$ in $L$. Thus by Lemma 2.2, $a$ has a supplement in $L$.

Using Theorem 2.4 we prove that if a lattice $L$ is an arbitrary join of cofinitely supplemented principal ideals, then $L$ is cofinitely supplemented.

Theorem 2.5 Let $\left\{a_{i} / 0\right\}_{i \in I}$ be a collection of cofinitely supplemented sublattices of $L$ with $1=\bigvee_{i \in I} a_{i}$. Then $L$ is a cofinitely supplemented lattice.

Proof Let $m$ be any maximal element of $L$. If $a_{i} \leq m$ for all $i \in I$, then $1=\bigvee_{i \in I} a_{i} \leq m$ which is a contradiction. So there exists a $j \in I$ such that $a_{j} \not \leq m$. Then $1=a_{j} \vee m$. Since $a_{j} /\left(a_{j} \wedge m\right) \cong\left(a_{j} \vee m\right) / m=1 / m$, the element $a_{j} \wedge m$ is maximal in $a_{j} / 0$. By hypothesis there is a supplement $c$ of $a_{j} \wedge m$ in $a_{j} / 0$, i.e. $\left(a_{j} \wedge m\right) \vee c=a_{j}$ and $a_{j} \wedge m \wedge c \ll c / 0$. If $c \leq m$, then $a_{j}=\left(a_{j} \wedge m\right) \vee c \leq m$, a contradiction. So $c \not \leq m$. Therefore, $1=m \vee c$ and $m \wedge c=a_{j} \wedge m \wedge c \ll c / 0$. Thus $c$ is a supplement of $m$ in $L$. By Theorem 2.4, $L$ is a cofinitely supplemented lattice.

Theorem 2.4 is also used in the proof of the following theorem which gives a new result for modules:

Theorem 2.6 If a/0 is a cofinitely supplemented sublattice of $L$ and $1 /$ a has no maximal element, then $L$ is also a cofinitely supplemented lattice. 
Proof Let $m$ be a maximal element of $L$. If $a \leq m$, then $m$ is a maximal element of $1 / a$, but $1 / a$ has no maximal element. So $a \not \leq m$; therefore $a \vee m=1$ and $a /(a \wedge m) \cong(a \vee m) / m=1 / m$. Since $m$ is a maximal element of $L, a \wedge m$ is a maximal and therefore a cofinite element of $a / 0$. Then there is a supplement $c$ of $a \wedge m$ in $a / 0$, that is $(a \wedge m) \vee c=a$ and $(a \wedge m) \wedge c \ll c / 0$. Since $c$ is in $a / 0, c \wedge m=c \wedge(a \wedge m) \ll$ $c / 0 . c \vee m=c \vee(a \wedge m) \vee m=a \vee m=1$. So $c$ is a supplement of $m$ in $L$. By Theorem 2.4, $L$ is a cofinitely supplemented lattice.

For a module $K$ over a ring the radical $\operatorname{Rad} K$ of $K$ is the intersection of all maximal submodules of $K$, so $\operatorname{Rad} K=K$ if $K$ has no maximal submodules.

Corollary 2.7 Let $M$ be a module, $N$ be a cofinitely supplemented submodule of $M$. If $\operatorname{Rad}(M / N)=M / N$, then $M$ is cofinitely supplemented.

\section{Amply Supplemented Lattices}

A homomorphic image of a small element under a lattice morphism need not be small unlike the module case [2, Example 2.1]. Nevertheless, we will show that the quotient sublattices $1 / a$ of an amply supplemented lattice is amply supplemented by using properties of small elements given in Lemma 1.1.

Proposition 3.1 If a lattice $L$ is amply supplemented, then for every element a of $L$ the quotient sublattice $1 / a$ is amply supplemented.

Proof Let $x$ be an element of $1 / a$. If $x \vee y=1$ for some $y \in 1 / a$, then $x$ has a supplement $y^{\prime} \leq y$ in $L$ because $L$ is amply supplemented, i.e. $x \vee y^{\prime}=1$ and $x \wedge y^{\prime} \ll y^{\prime} / 0$. Then $x \vee\left(y^{\prime} \vee a\right)=1$. By modular law, $x \wedge\left(y^{\prime} \vee a\right)=a \vee\left(x \wedge y^{\prime}\right)$ and since $x \wedge y^{\prime} \ll y^{\prime} / 0, a \vee\left(x \wedge y^{\prime}\right) \ll\left(y^{\prime} \vee a\right) / a$ by Lemma 1.1 (1). So $y^{\prime} \vee a$ is a supplement of $x$ in $1 / a$ with $y^{\prime} \vee a \leq y$.

Proofs of Proposition 3.2 and 3.4 are similar to the proofs of [7, 41.7(1)] and [7, 41.8].

Proposition 3.2 If $L$ is an amply supplemented lattice, then for every supplement a of an element of $L, a / 0$ is amply supplemented.

If $a \vee a^{\prime}=1$ and $a \wedge a^{\prime}=0$ for elements $a$ and $a^{\prime}$ of $L$, then we use the notation $a \oplus a^{\prime}=1$ and call this a direct sum. In this case $a$ and $a^{\prime}$ are called direct summands of 1 .

Corollary 3.3 [7, 41.7(2)] If L is amply supplemented, then for a direct summand a of $L$, the quotient sublattice a/0 is amply supplemented.

Proposition 3.4 Let $a, b \in L$ with $a \vee b=1$. If $a$ and $b$ have ample supplements in $L$, then $a \wedge b$ has also ample supplements in $L$.

Given elements $a \leq b$ of $L$, the inequality $a \leq b$ is called cosmall in $L$ if $b \ll 1 / a$. One can easily modify the proofs of $[8$, Proposition 2.1] and $[4,20.24]$ to prove the following proposition:

Proposition 3.5 The following are equivalent for a lattice $L$ :

(a) L is amply supplemented.

(b) Every element $a$ of $L$ is of the form $a=x \vee y$ with $x / 0$ supplemented and $y \ll L$.

(c) For every element a of $L$, there is an element $x \leq$ a such that the quotient sublattice $x / 0$ is supplemented with the inequality $x \leq$ a cosmall in $L$.

Corollary 3.6 If the quotient sublattice a $/ 0$ is supplemented for every element a of a lattice $L$, then $L$ is amply supplemented.

Proposition 3.7 Let $L$ be an amply cofinitely supplemented lattice. Then for every a $\in L, 1 / a$ is amply cofinitely supplemented.

Proof Let $b$ be a cofinite element of $1 / a$. Then $1 / b$ is compact. So $b$ is a cofinite element of $L$. Suppose $b \vee c=1$ for some $c \in 1 / a$. Since $L$ is amply cofinitely supplemented, $c / 0$ contains a supplement $x$ of $b$ in $L$, i.e. $b \vee x=1$ and $b \wedge x \ll x / 0$. Then $b \vee(x \vee a)=1 \vee a=1$ and by Lemma 1.1 (1),

$$
(a \vee x) \wedge b=(b \wedge x) \vee a \ll(x \vee a) / a,
$$

i.e. $x \vee a$ is a supplement of $b$ in $1 / a$. Since $a \leq c$ and $x \leq c$, we have $a \vee x \leq c$. Hence, $1 / a$ is amply cofinitely supplemented. 
Lemma 3.8 If $a$ is a cofinite element of a lattice $L$, then there exists a maximal element $m$ of $L$ such that $a \leq m$.

Proof Since $a$ is a cofinite element of $L$, by [3, Lemma 2.4] there is a maximal element $m$ in $1 / a$. So $m$ is a maximal element of $L$ containing $a$.

The following theorem generalizes [1, Theorem 2.10] to lattices:

Theorem 3.9 The following are equivalent for a lattice $L$ :

(a) L is amply cofinitely supplemented.

(b) Every maximal element of $L$ has ample supplements in $L$.

(c) For every cofinite element $a$ and an element $b$ of $L$ with $a \vee b=1$ there exists an element $c=\bigvee_{i \in F} l_{i}$ where $F$ is a finite set and each $l_{i}$ is a local element of $b / 0$ such that $a \vee c=1$.

Proof $(a) \Rightarrow(b)$ Clear since every maximal element $m$ is cofinite.

(b) $\Rightarrow(c)$ Let $a$ be a cofinite element of $L, b \in L$ and $a \vee b=1$. Let

$$
C=\left\{c \in b / 0 \mid c=\bigvee_{i \in F} l_{i}, F \text { is finite, } l_{i} \text { is local }\right\} .
$$

Suppose that $a \vee c \neq 1$ for every $c \in C$. Let $\Omega$ denote the collection of elements $x$ of $L$ such that $a \leq x$ and $x \vee c \neq 1$ for every $c \in C$. Let $\Gamma=\left\{x_{\lambda} \mid \lambda \in \Lambda\right\}$ be a chain in $\Omega$ and $x=\bigvee_{\lambda \in \Lambda} x_{\lambda}$. Since $\forall \lambda, x_{\lambda} \in \Omega, a \leq x_{\lambda}$. Then $a \leq \bigvee_{\lambda \in \Lambda} x_{\lambda}=x$. So $x$ is an upper bound for $\Gamma$. Suppose that $\left(\bigvee_{\lambda \in \Lambda} x_{\lambda}\right) \vee c=1$ for some $c \in C$. Since $1 / a$ is compact, there exists a finite subset $F$ of $\Lambda$ such that $x=\left(\bigvee_{\lambda \in F} x_{\lambda}\right) \vee c=1$. Then $\bigvee_{i \in F} x_{\lambda}=x_{\lambda_{0}}$ for some $\lambda_{0} \in F$, so $x_{\lambda_{0}} \vee c=1$. This is a contradiction. Thus $x=\bigvee_{\lambda \in \Lambda} x_{\lambda} \in \Omega$. By Zorn's Lemma $\Omega$ contains a maximal element $u \neq 1$. Since $a$ is cofinite, $1 / a$ is compact and since $a \leq u, 1 / u$ is compact, i.e. $u$ is cofinite. By Lemma 3.8, there exists a maximal element $m$ of $L$ such that $u \leq m$ and $m \vee b=1$. Since $m$ has ample supplements, there exists $y \in b / 0$ such that $y$ is a supplement of $m$ in $L$. By Lemma 2.3, $y / 0$ is local. Since $y\lfloor\leq m, y \not \leq u$. Therefore, $u \neq u \vee y$. By maximality of $u$, there exists an element $v \in C$ such that $1=(u \vee y) \vee v$. Since $y \vee v \leq b$ and $y \vee v$ is a finite join of local elements, $1=u \vee(y \vee v)$, therefore $u \notin \Omega$. This is a contradiction.

$(c) \Rightarrow(a)$ Suppose that $a$ is a cofinite element and $a \vee b=1$ for some $b \in L$. We want to show that $a$ has ample supplements in $b / 0$. By hypothesis there is an element $c=\bigvee_{i \in F} l_{i}$ where $F$ is a finite set and each $l_{i}$ is a local element of $b / 0$ such that $a \vee c=1$. Since 0 is a supplement of $1=a \vee c$, there exists a subset $J \subseteq F$ such that $\bigvee_{i \in J} l_{i}$ is a supplement of $a$ in $b / 0$ by Lemma 2.2.

Clearly, every element of a compact lattice is a cofinite element. So we have the following corollary:

Corollary 3.10 A compact lattice $L$ is amply supplemented if and only if every maximal element has ample supplements in $L$.

Acknowledgments The authors would like to thank the referees for their valuable suggestions and comments.

\section{References}

1. Alizade, R.; Bilhan, G.; Smith, P.F.: Modules whose maximal submodules have supplements. Commun. Algebra 29(6), 2389-2405 (2001)

2. Alizade, R.; Toksoy, S.E.: Cofinitely weak supplemented lattices. Indian J. Pure Appl. Math. 40(5), 337-346 (2009)

3. Cǎlugăreanu, G.: Lattice Concepts of Module Theory. Kluwer, Dordrecht (2000)

4. Clark, J.; Lomp, C.; Vanaja, N.; Wisbauer, R.: Lifting Modules. Supplements and Projectivity in Module Theory, Frontiers Mathematics. Birkhäuser, Basel (2006)

5. Çetindil, Y.: Generalizations of Cofinitely Supplemented Modules to Lattices. M.Sc. thesis, Izmir Institute of Technology (2005)

6. Stenström, B.: Radicals and socles of lattices. Arch. Math. 20, 258-261 (1969)

7. Wisbauer, R.: Foundations of Module and Ring Theory. Gordon and Breach, Philadelphia (1991)

8. Zöschinger, H.: Komplemente als direkte Summanden. Arch. Math. 25, 241-253 (1974) 